\title{
11
}

\section{Dossier de Progression et Dossier de Réussite: Deux Outils Complémentaires au Service de la Professionnalisation Enseignante dans le Supérieur}

\author{
Marianne Poumay et François Georges \\ Université de Liège, Belgique
}

Cet article aborde et illustre les notions de référentiel de compétences, de dossier de progression et de dossier de réussite (Tardif, 2006) tels quils sont mis en cuvre au sein du programme Formasup, master complémentaire en pédagogie de l'enseignement supérieur organisé à l'université de Liège. Les auteurs présentent l'articulation interne de ce programme qui cible un nombre limité de compétences critiques, accompagne le développement de ces compétences chez les enseignants participants et mesure ce développement à travers un portfolio. Ils discutent enfin des défis et difficultés qui persistent ainsi que des moyens mis en place par l'équipe encadrante pour tenter de les surmonter.

\section{Introduction}

$\mathrm{D}$

ans l'enseignement supérieur, nombreux sont les dispositifs de formation innovants, qui tentent d'harmoniser les intentions et les actions, renforçant la cohérence entre les compétences visées, les méthodes mises en ouvre pour développer ces compétences et la façon dont ce développement de compétences sera évalué. Cette " triple concordance " entre objectifs (parfois rédigés en termes de compétences), méthodes et évaluation - qui favorise l'apprentissage et, en corollaire, la réussite des étudiants - est déjà complexe à installer au niveau d'un cours. Elle l'est encore davantage au niveau d'un pro- gramme entier de formation.

"Formasup ", le master complémentaire en pédagogie de l'enseignement supérieur de l'ULg, tente de mettre en pratique cette concordance entre visées et outils.

\section{Les principes de Formasup}

Dans Formasup, les enseignants du cycle supérieur sont en réflexion sur leur pratique. Chacun mène son propre projet, vit des situations d'apprentissage 
variées, bénéficie de supports en ligne et participe à deux semaines de regroupement présentiel par an, ceci durant deux années. Tout au long de ces deux années, même si les rencontres sont rares, la progression est très encadrée grâce à des " accompagnateurs " individuels (pour plus de détails sur cette formation, voir Poumay 2006 ainsi que le site informatif ci-dessous).

Le master est fondé sur les principes du " Scholarship of Teaching and Learning " (SoTL, Kreber 2002) : les enseignants participants sont appelés à documenter leur pratique et à innover dans leur propre enseignement en y menant une réelle recherche. Celle-ci nécessite des analyses de données subjectives (ex. analyse des avis de leurs étudiants) mais aussi objectives (ex. analyse des notes obtenues pour des travaux, traces d'apprentissage en ligne), et doit impérativement servir l'apprentissage de leurs étudiants. Ils sont également tenus de communiquer sur leur pratique, de partager leurs réflexions avec leurs collègues et d'ainsi contribuer à la diffusion des savoirs après avoir contribué à leur construction.

Formasup est aussi ancré dans le courant de l'apprentissage visant le développement de compétences. Il a été fortement affecté en 2006 par l'ouvrage de Jacques Tardif qui propose une définition opérationnelle de la compétence et décrit des outils (portfolios, cartes conceptuelles) qui facilitent l'évaluation des étudiants en cohérence avec cette définition. Nous nous en sommes fortement inspirés pour revisiter notre référentiel et consolider notre approche.

\section{Les Outils Déployés}

Comme l'illustre la figure suivante, basé sur un référentiel de neuf compétences à développer chez les

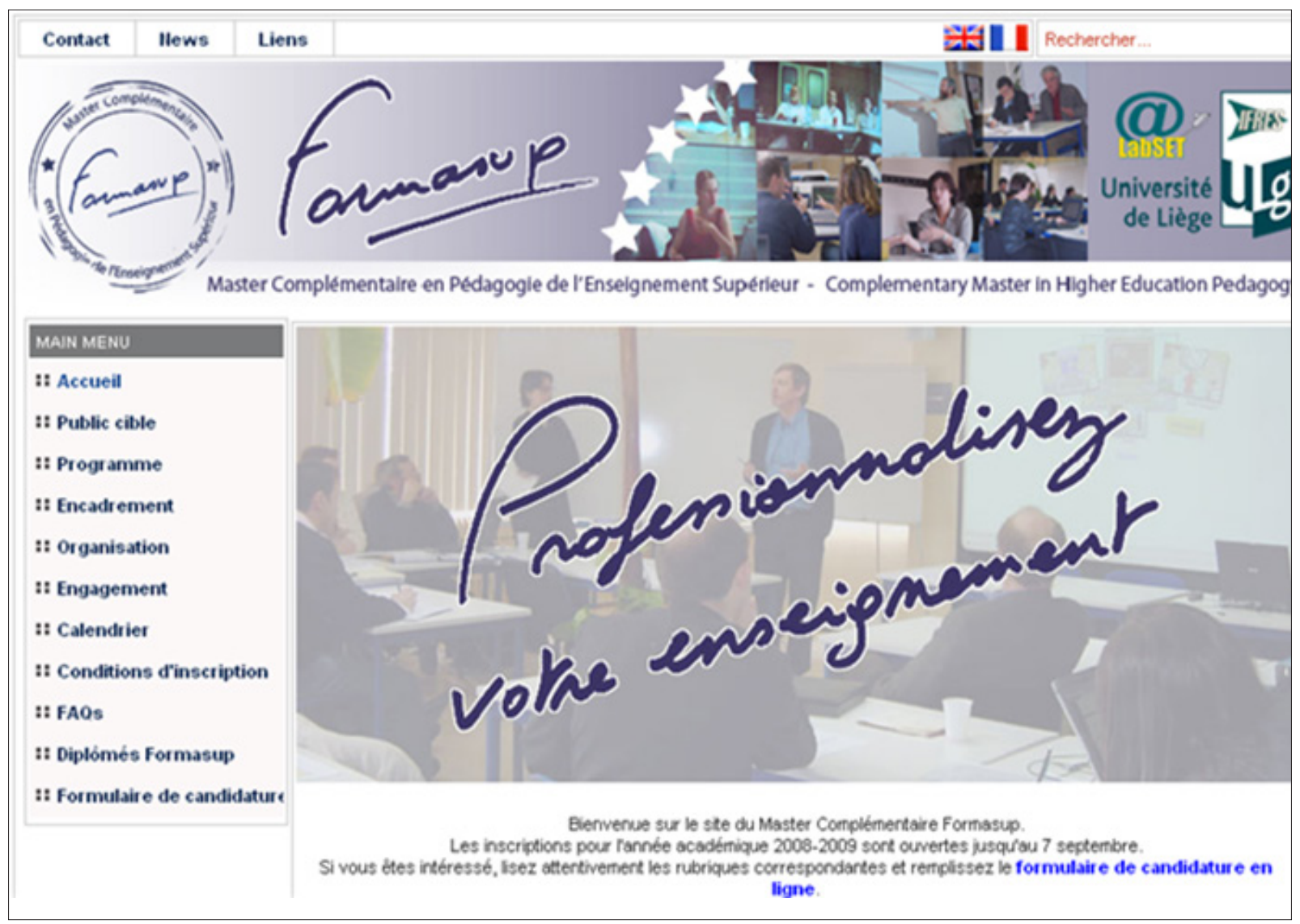

Figure 1

Le site de présentation du master complémentaire Formasup, disponible à l'adresse http://www.formasup.eu 
enseignants participants, Formasup met en œuvre un « dossier de progression » (Tardif, 2006) pour accompagner ces enseignants de façon étroite et un « dossier de réussite " pour évaluer le développement des neuf compétences visées.

Le référentiel reprend neuf des compétences inhérentes à la professionnalisation enseignante, qui sont développées au sein de Formasup. D’autres actions de l'enseignant mériteraient sans doute tout autant notre attention mais nous nous limitons à ce que nous pensons pouvoir développer et mesurer. Ces neuf compétences développées appartiennent aux domaines conceptuel (ex. formaliser ses savoirs d'expérience), pratique (ex. réguler en menant une recherche située) et réflexif ou relationnel (ex. faire preuve d'esprit critique et développer celui-ci chez ses étudiants). Elles résultent de la documentation de nos propres pratiques, mais aussi de nos régulations annuelles et de nos réflexions quant à la cohérence de nos outils. En effet, par exemple, pour répondre à la définition que donne Tardif (2006) de la compétence, il nous faut rendre possible pour chacune de nos neuf compétences une multiplicité de parcours d'apprentissage différents, une mobilisation et une combinaison de ressources propre à chaque enseignant participant, différente de celle de son collègue, et un apport de preuves du développement de cette compétence. Les compétences doivent en outre être

\section{Un référentiel (pour formaliser 9 compétences à développer)}

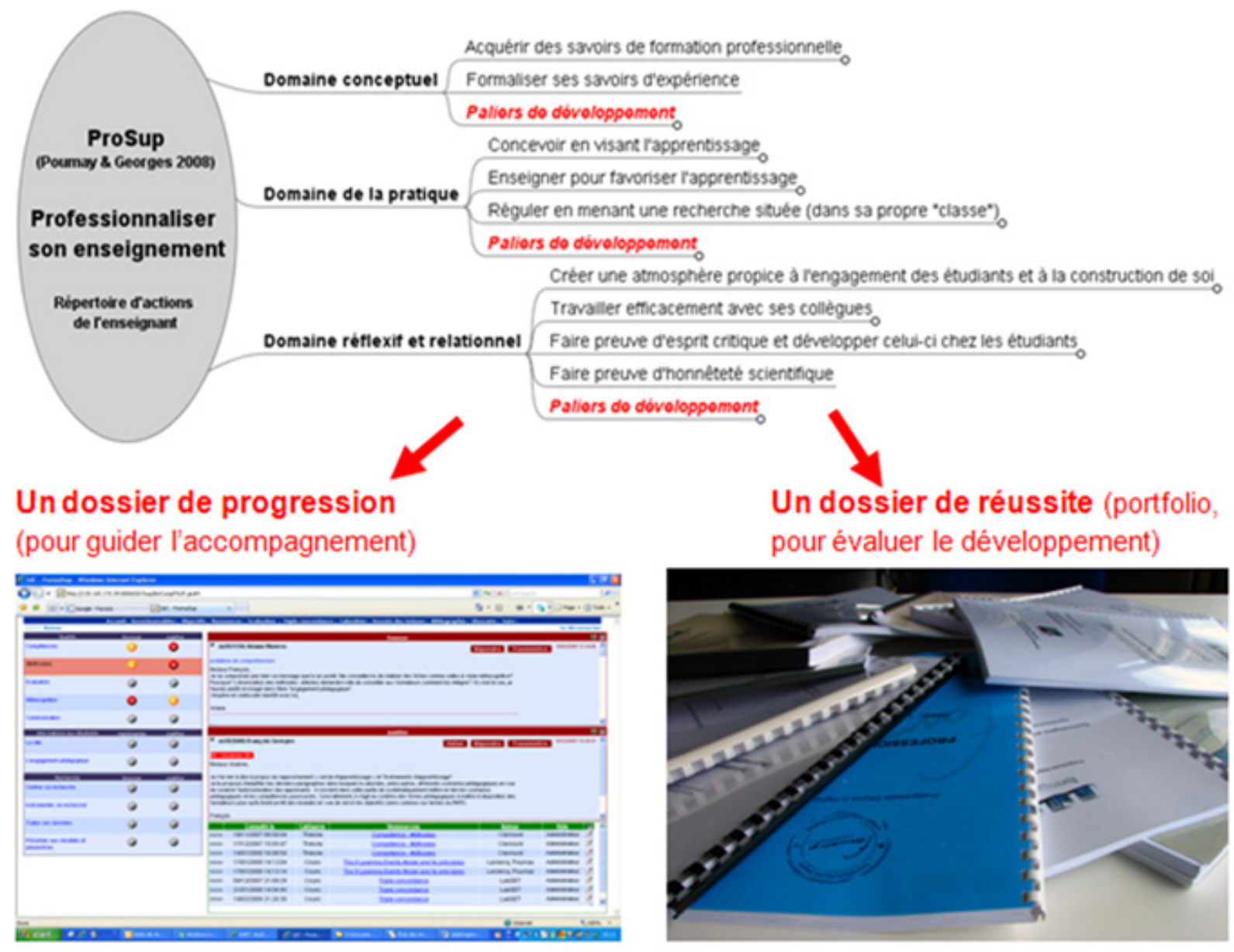

Figure 2

Un référentiel et deux outils pour guider et évaluer le développement professionnel des enseignants du supérieur 
considérées comme critiques dans la profession enseignante. La plupart des compétences (voir ci-dessous l'exemple de la compétence " concevoir en visant l'apprentissage »), sont elles-mêmes décomposées en des constituants plus fins, de façon à baliser la progression des enseignants participants.

Pour soutenir le développement de ces compétences, le «dossier de progression » documente le parcours de développement de plusieurs d'entre elles. Il permet à chaque participant de dialoguer avec un accompagnateur personnel tout au long de la conception de la nouvelle mouture de son cours (au sein de son groupe classe) et de sa question de recherche. Ce dossier est informatisé, le dialogue est donc instrumentalisé. L'organisation asynchrone est adaptée aux contraintes des enseignants participants. Dans ce dossier interactif, l'enseignant participant communique des preuves de son degré de maîtrise des nombreuses ressources propre à la formation. Pour structurer son travail, une dizaine d'items ont été isolés et illustrés de théorie et d'exemples. Son accompagnateur lui fournit sur sa production autant de feed-back qu'il le souhaite. La fonction principale de cet accompagnement consiste en un soutien à l'apprentissage mais la fonction de soutien affectif des participants est elle aussi bel et bien présente.

En organisant une auto-évaluation permanente et assistée, cet outil d'accompagnement constitue un puissant levier de prise de conscience par chacun de son apprentissage. Par ailleurs, par l'aspect diagnostic des interactions avec les accompagnateurs, l'outil fournit à chacun des données utiles à son autorégulation permanente.

En fin d'année, le "dossier de réussite " rassemble les preuves du développement professionnel de chaque enseignant. Il demande à l'enseignant participant de décrire son parcours en tant qu'enseignant, de souligner les valeurs qu'il véhicule ainsi que les grands tournants pédagogiques de sa carrière, de décrire son action innovante et la recherche qu'il a menée au service de l'apprentissage de ses étudiants, de fournir des preuves de cinq critères de qualité de son enseignement (suivant les " Prompts for good practice » de l'association HERDSA) et de décrire les perspectives de son développement professionnel.

Ce portfolio fait l'objet d'une évaluation par trois membres d'un jury. Les décisions quant au degré de maîtrise des ressources et au niveau de développement de chaque compétences se prennent donc collégialement. L'accompagnateur de chaque participant ne

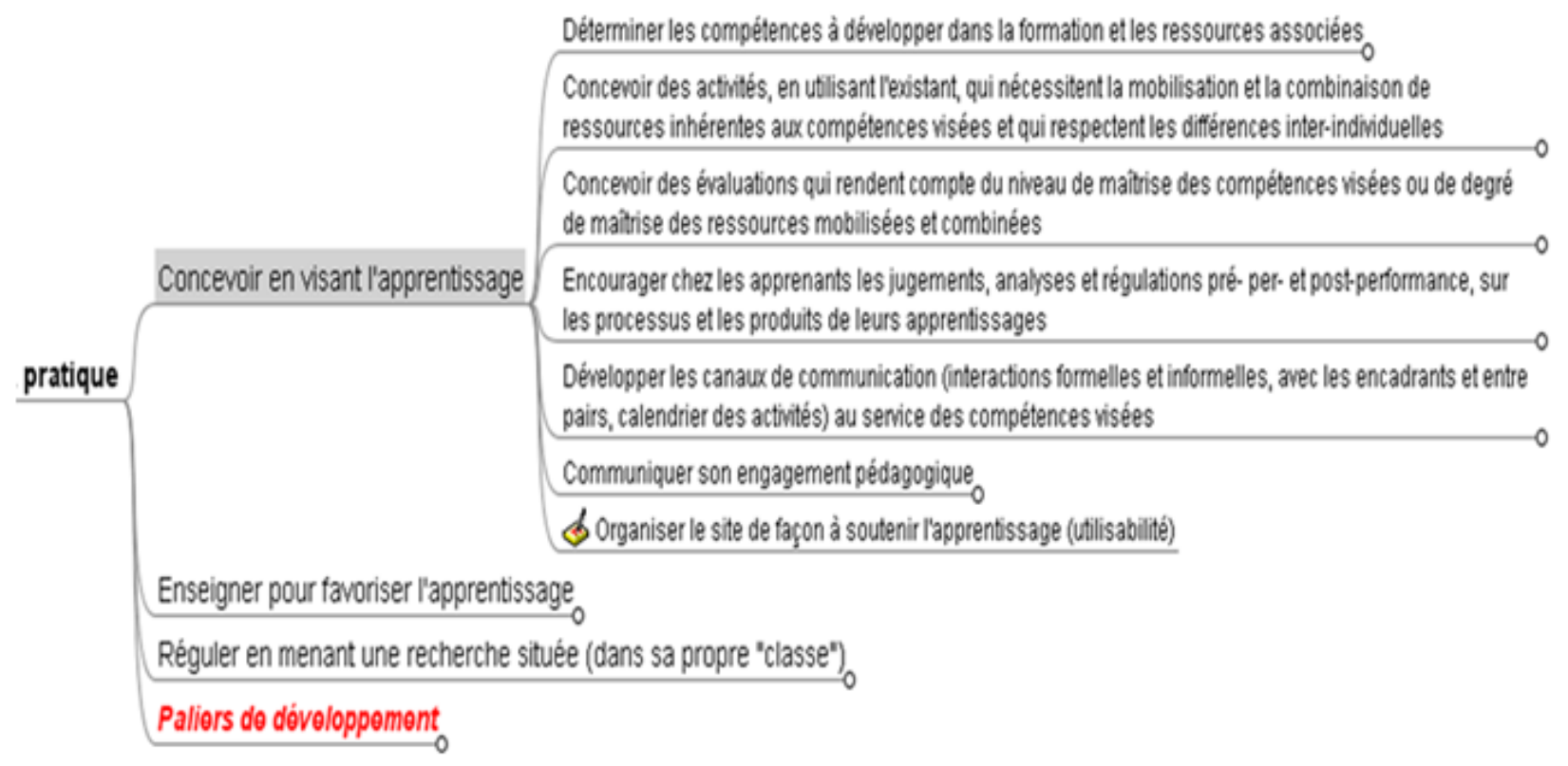

Figure 3

Décomposition de la compétence " concevoir en visant l'apprentissage » 


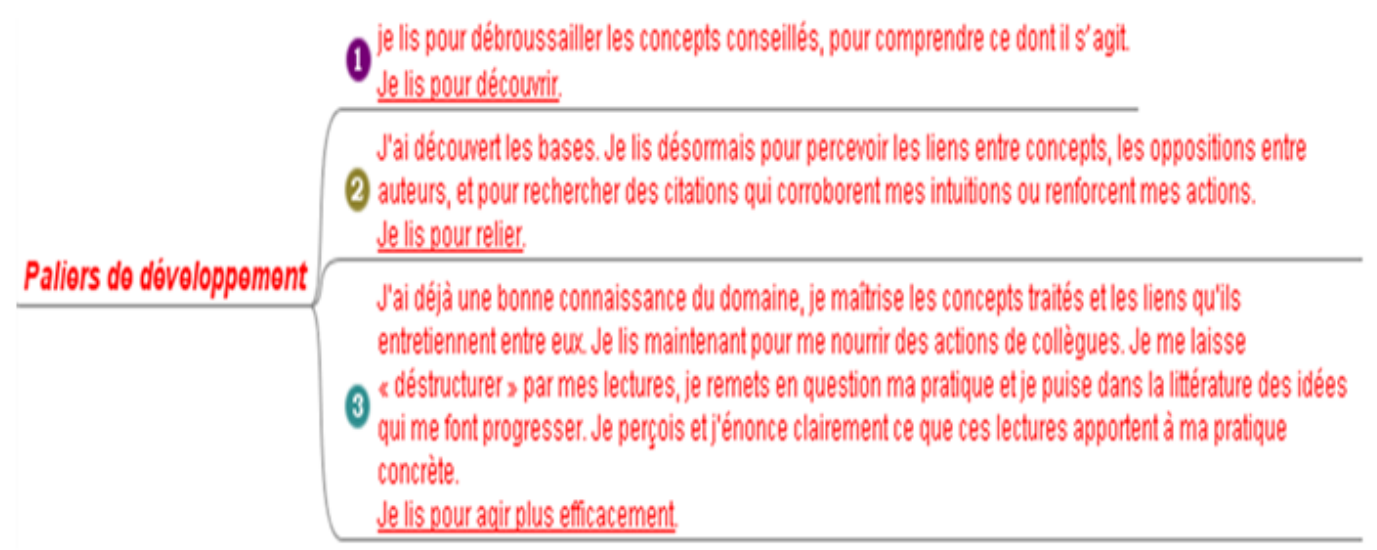

Figure 4

Première tentative de définition de paliers de développement pour les compétences du domaine conceptuel

fait pas partie de ces trois juges mais il est présent lors de la délibération et fait parfois valoir des arguments en faveur du participant. De type holistique et orienté recherche, le portfolio vise pourtant également à valoriser l'enseignant participant en lui offrant la possibilité de communiquer sur sa pratique. La constitution de ce dossier est largement facilitée par le parallélisme entre les deux instruments : progression et réussite portent sur une série de critères communs repris dans le référentiel de compétences, fil rouge de la formation.

\section{Discussion et Perspectives}

Vu l'exigence de ce diplôme, qui demande à chaque enseignant un considérable investissement en temps et en énergie ainsi qu'une performance finale (le portfolio, ou dossier de réussite) d'un niveau très élevé, deux ingrédients nous semblent indispensables : un accompagnement très étroit et une cohérence du programme.

L'accompagnement, comme nous l'avons souligné plus haut, constitue à la fois un soutien à l'apprentissage et un soutien affectif. Individuel et rapproché, il est d'ailleurs considéré par les participants comme l'un des points forts du programme. La cohérence entre le référentiel, le dossier de progression et le dossier de réussite est absolument nécessaire pour que les enseignants perçoivent l'intérêt des tâches à réaliser, des séminaires proposés et des ressources présentes en ligne. Ces professionnels qui ont un emploi d'enseignant à temps plein ne peuvent dégager du temps que s'ils sont convaincus de l'intérêt de ces efforts pour la qualité de leur enseignement. La cohérence de notre offre de formation doit être rapidement perçue par chacun, de façon à favoriser la persévérance.

Cette cohérence a beau se renforcer d'année en année, l'architecture de base du programme a beau nous sembler fondée sur des principes aujourd'hui bien documentés en pédagogie universitaire (SoTL, compétences, professionnalisation enseignante), des progrès restent à faire et notre équipe encadrante est toujours en réflexion. Nous constatons par exemple une difficulté récurrente de nos participants à toujours bien sélectionner leurs actions en fonction de leur sens. Choisir de mener une action qui répond réellement à l'une de leurs difficultés en classe, mener une recherche qui serve effectivement l'apprentissage de leurs étudiants, orienter leur réflexion vers les éléments les plus critiques de leur pratique sont autant d'actions qui sont loin d'être intuitives chez tous les enseignants.

Pour les aider dans cette voie, nous aimerions fournir aux enseignants des "paliers ", des niveaux de développement de chacune des compétences ciblées par notre référentiel. Ces niveaux prendraient clairement en compte cette notion de sens des actions, tellement importante en développement professionnel. 
Nous voyons ces niveaux de développement comme autant de guides à l'auto-estimation des progrès des enseignants.

Ces paliers sont malheureusement très complexes à établir. Nous nous y attachons, nous en approchons certains, mais il nous faudra sans doute encore plusieurs années pour déterminer précisément ces repères qui serviraient l'accompagnement et le développement. A titre d'illustration, la Figure 4 reprend une première ébauche de trois paliers successifs, pour les compétences appartenant au domaine conceptuel.

Pour établir ces paliers, nous avons analysé les dossiers de progression et les dossiers de réussite des deux dernières cohortes d'enseignants de Formasup. Depuis 2008, nous ajoutons à ces analyses un questionnement des participants qui, à quatre reprises sur l'année, nous donnent des indications sur leur perception de paliers critiques dans leurs compétences et des éléments qui ont été les plus déclencheurs de ces sauts qualitatifs. Notre hypothèse est que connaître ces paliers va aider les enseignants à prendre conscience, puis à se rapprocher de ce que nous considérons comme le niveau le plus élevé de professionnalisation enseignante au regard des quelques compétences sélectionnées.

Sans doute des collaborations internationales entre équipes constitueraient-elles un facteur d'accélération de ces réflexions et de qualité de leurs résultats. Il serait utile de comparer tant nos méthodes de recueil de données que nos résultats - les paliers - et la façon dont nous en tirons parti pour améliorer nos programmes.

\section{Références}

Kreber, C. (2002). Teaching excellence, teaching expertise, and the scholarship of teaching. Innovative Higher Education, 27(1), 5-23. Récupéré le 17 octobre 2006 du site de la revue, http://springerlink.metapress.com/content/ g4p670700rv77017/fulltext.pdf

Poumay, M. (2006). Pour professionnaliser le métier d'enseignant du supérieur : le Master Com- plémentaire Formasup. Revue internationale des technologies en pédagogie universitaire, 3(1), p. 3-13. Article disponible en ligne à l'adresse http://www.profetic.org/revue/IMG/pdf/ RITPU_Poumay01_03-01.pdf

Tardif, J. (2006). L'évaluation des compétences. Documenter le parcours de développement. Montréal : Chenelière Education.

\section{Biographies}

Marianne Poumay est professeure à l'université de Liège et directrice du LabSET-ULg. Cette équipe de 35 personnes étudie l'apprentissage, en particulier dans un contexte d'usage de technologies. Marianne est à l'initiative du master complémentaire Formasup, qu'elle s'attache à faire évoluer au fil des ans, tel un laboratoire de principes et méthodes d'accompagnement des enseignants dans leur professionnalisation.

François Georges est directeur adjoint du LabSET (IFRES - Université de Liège). Il intervient dans le master complémentaire en pédagogie de l'enseignement supérieur dans lequel il accompagne les inscrits dans la conception, l'implémentation, l'évaluation et la régulation de leur dispositif de formation. François poursuit un doctorat sur l'identification et la maximisation des parcours de professionnalisation des enseignants. 